\title{
Efficient Routing Protocol for Hand over Delay Minimization in Mobile WiMAX using Concurrent Scanning
}

\author{
Arathi R Shankar \\ Associate Professor \\ Department of ECE,BMSCE \\ Bangalore, India
}

\author{
Prabhu Raddy \\ Department of ECE \\ BMSCE \\ Bangalore, India
}

\author{
V. Sambasiva Rao \\ Professor \\ Department of ECE, PESIT \\ Bangalore, India
}

\begin{abstract}
In this article an efficient routing protocol has been identified for real time and non - real time applications. The MS movement direction prediction (MMDP) based MS scanning is implemented to overcome the mobile WiMAX handover issues. Here the authors have used the MMDP method to reduce the number of scanning's required for the handover instead of scanning for all neighboring BSs and suggested that on using the concurrent scanning procedure for the best two TBS high quality hand over support in Mobile WiMAX can be achieved. On performing concurrent scanning of two best target base stations, issues like ideal sectors, network congestion and fast change in RSS are proposed to be minimized.The proposed algorithm targets to meet the quality of service requirements. Performance results for simulation scenarios are presented and discussed.
\end{abstract}

\section{Keywords}

Routing protocols, MMDP scanning, concurrent scanning.

\section{INTRODUCTION}

Mobile WiMAX is an enhanced version of IEEE 802.16 standard with mobility support. It offers scalability and supports flexible network architecture. Amendments support scalable channel bandwidths from 1.25 to $20 \mathrm{MHz}$ [1]. Mobile WiMAX IEEE 802.16e supports mobility and is also capable to provide fixed access. It adopts Orthogonal Frequency Division Multiple Access (OFDMA) for improved multi-path performance in non-line-of-sight environments. The Mobile WiMAX profiles will cover 5, 7, 8.75, and $10 \mathrm{MHz}$ channel bandwidths for licensed worldwide spectrum allocations in the $2.3 \mathrm{GHz}, 2.5 \mathrm{GHz}, 3.3 \mathrm{GHz}$ and $3.5 \mathrm{GHz}$ frequency bands [2]. Features of IEEE 802.16e are Scalable OFDMA, Advanced antenna diversity schemes, hybrid automatic repeat-request (HARQ), Adaptive Antenna Systems (AAS), MIMO technology. Turbo Coding and Low-Density Parity Check (LDPC) are introduced. Automatic Repeat request (ARQ) or Forward Error Correction (FEC) are the error correction techniques that are adopted. A combination of both the above mentioned methods is called Hybrid ARQ (HARQ).

\section{HAND OVER}

\subsection{Methodology:}

Hand over is required in mobile wireless communication to maintain uninterrupted services during user's movement from one location to another. This can affect the QoS \& capacity of mobile broadband network. The hand over techniques can be divided into soft hand over (make_ before_ break) \& hard over (break_before_make). In Soft hand over connection, the serving base station (SBS) is terminated before a mobile station(MS) switches to another base station. Break time is zero during hand over and spectral efficiency is reduced.. Its counterpart Hard Handover_(HHO) uses a break before make approach. Mobile WiMAX network uses packet switching with mostly bursty delay tolerant data traffic. Therefore HHO is used in mobile WiMAX.

The intersection between the two received signal strength (RSS) lines is the criteria for Hand off (HO) trigger. Frequent $\mathrm{HO}$ should be avoided, and a $\mathrm{HO}$ is triggered only when the difference between RSS of serving Base Station (BS) and target BS (TBS) is below the target value. Handover takes place in two main phases; the network topology acquisition, also known as pre-handover phase, and the actual handover phase. The first phase includes network topology advertisement, neighboring Base Station (nBS) scanning and association. The second phase includes handover decision and initiation, and network re-entry synchronization and ranging with a TBS. In the scanning process, the MS must synchronize with all advertised (nBS) to select the best BS candidate for the incoming handover action. Without terminating the connection between the Serving Base Station (SBS) and Mobile Station (MS), the SBS will schedule the scanning intervals or sleep-intervals to MS for scanning.

\subsection{MMDP Scheme}

The SBS keeps track of the MS movement location and trajectory based on LBS as it is supported in the new WiMAX standard of IEEE $80216 \mathrm{~m}$. Based on [10], the BS coverage area is divided into zones and sectors as shown in Figure 1. The hexagonal BS structure is divided into three zones: no handover (No-HO) zone, low handover (Lo-HO) zone and high handover (High-HO) zone based on signal quality [8]. The No-HO zone is considered as no handover probability, while Lo-HO and High-HO zones are considered as low to high handover probabilities. This division subsequently reduces the actual area of MS's random movement tracking during a potential handover activity. Also, the cell structure is divided into six sectors to simplify the SBS calculation of the distance between MS and nBSs. Unlike [9], the SBS will utilize this information to calculate the distance between MS and only to the $\mathrm{n}$ BSs which located in the same sector as the current MS location where the neighboring sectors will be considered on distance calculation process. As shown in Figure 1 where only BSs B, C and D are considered, the SBS in the Lo-HO zone will start to predict the MS movement direction. 
The hexagonal BS structure of Fig 1 is divided into three zones: no handover (No-HO) zone, low handover (Lo-HO) zone and high handover (High-HO) zone. This sectorization is done based on signal strength [8]. The No-HO zone is considered as no handover probability, while Low-HO and High-HO zones are considered as low to high handover probabilities. This sectorization reduces the unnecessary random movement of MS's during handover activity. Also, the cell structure is divided into six sectors to simplify the SBS calculation of the distance between MS and nBSs. Due to the above method of sectorization, the SBS will calculate the distance between MS and the n BSs which are located in the same sector as the current MS location and the neighbouring sectors will be considered on distance calculation process [9]. As an example in Figure (1) where only BSs B, C and D are considered, the SBS in the Lo-HO zone will start to predict the MS movement direction[12].

The SBS calculates the distance between the MS and three potential TBSs of B, C and D as shown in Fig(1).Depending on their relative distance with MS, only the BS B and C are considered as a target base stations because their relative distances are less compared to BS D. One of the Base stations either $\mathrm{B}$ or $\mathrm{C}$ will become the serving base station. In this way we can reduce the number of scanning's in the pre hand over process [10].

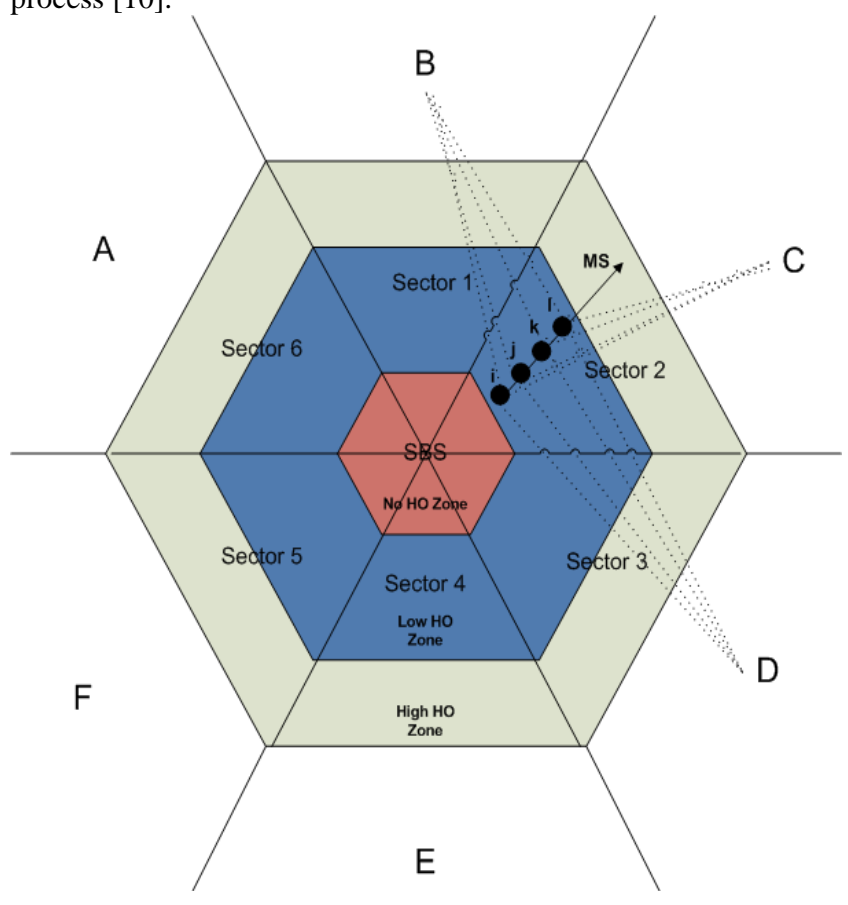

Fig. 1 MS movement prediction

\subsection{ROUTING PROTOCOLS}

\section{AODV}

Ad hoc On-Demand Distance Vector (AODV) Routing is a routing protocol for mobile ad hoc networks (MANETs) and other wireless ad-hoc networks. It is a reactive routing protocol, meaning that it establishes a route to a destination only on demand[13]. In contrast, the most common routing protocols of the Internet are proactive, meaning they find routing paths independently of the usage of the paths. The advantage of AODV is that it creates no extra traffic for communication along existing links. Also, distance vector routing is simple, and doesn't require much memory or calculation. However AODV requires more time to establish a connection, and the initial communication to establish a route is heavier than some other approach.

\section{Bellman-Ford}

The Bellman-Ford algorithm is an algorithm that computes shortest paths from a single source vertex to all of the other vertices in a weighted digraph. It is slower than Dijkstra's algorithm for the same problem, but more versatile, as it is capable of handling graphs in which some of the edge weights are negative numbers. In such a case, the Bellman-Ford algorithm can detect negative cycles and report their existence, but it cannot produce a correct "shortest path" answer if a negative cycle is reachable from the source, because there is no well-defined "shortest path" in that case.

\section{DYMO}

The DYMO routing protocol is successor to the popular Ad hoc On-Demand Distance Vector (AODV) routing protocol and shares many of its benefits. It is, however, slightly easier to implement and designed with future enhancements in mind. This can work as both a pro-active and as a reactive routing protocol, i.e. routes can be discovered just when they are needed.

\section{FSR}

Fisheye State Routing (FSR) belongs to the class of proactive (table-driven) ad hoc routing protocols and its mechanisms are based on the Link State Routing protocol used in wired networks. It tries to minimize the routing overhead by using a fisheye technique. Each node assigns other network participants to specific fisheye scopes dependent on their distance to the node itself. The amount of routing information is reduced by assuming longer link-state update intervals for nodes at higher distances than for network participants in the node's vicinity. Thus, FSR is intended to scale well in large mobile ad hoc networks and supports high rates of mobility.

\section{IERP}

This document describes the Inter-zone Routing Protocol (IERP), the reactive routing component of the Zone Routing Protocol (ZRP). IERP adapts existing reactive routing protocol implementations to take advantage of the known topology of each node's surrounding R-hop neighborhood (routing zone).When a global route discovery is required, the routing zone based broadcast service can be used to efficiently guide route queries outward, rather than blindly relaying queries from neighbor to neighbor. Once a route has been discovered, IERP can use routing zones to automatically redirect data around failed links.

\section{LANMAR}

LANMAR combines the features of Fisheye State Routing (FSR) and Landmark routing. This routing protocol is used for Large Scale Wireless Ad Hoc Networks with Group Mobility. It provides efficient and scalable routing in large, mobile, ad hoc environments in which group mobility applies. The Landmark Ad-hoc Routing Protocol (LANMAR) is designed 
to dramatically reduce routing table size and routing update overhead in large-scale ad-hoc networks that exhibit group mobility.

\subsection{Related Work}

The MS movement direction prediction (MMDP) based MS scanning is used to overcome the mobile WiMAX handover issues [11]. Here the authors propose to reduce the number of scanning's required for the handover instead of scanning for all neighbors BSs. On doing this there is a reduction in the total scanning delay and scanning interval duration.

\subsection{Proposed work}

On using the concurrent scanning procedure for best two target BSs high quality hand over support in mobile WiMAX can be achieved. On performing concurrent scanning of two best target base stations, issues like ideal sectors, network congestion and fast change in RSS are proposed to be minimized.

\section{Concurrent scanning operation}

After detecting the two best possible target base stations from the accumulative distances we consider the concurrent scanning for both the TBSs. On doing this we proposed to reduce the time duration required for scanning. Hence we are able to achieve the reduced pre hand over delay. Concurrent scanning simulation is done on the Matlab. Wherein we have proposed to use the two scanning modules for each TBSs. The counter and clocks used for them will be different.

Individual threads are created for both the modules. These are initiated scanning process is activated. In this operation one thread takes the op-code fetch and control switches to another thread where in it will start op-code fetching of second thread at this stage first thread goes to decoding the op-code. In this way simultaneous scanning operations are done for both the TBSs. The switching from one thread to another can take additional delay but that could be of very small compare to the scanning process.

\subsection{SIMULATION RESULTS}

A WiMAX Scenario consisting of two base stations and four mobile stations with all the parameters as per the IEEE 802.16e standard is modeled using Qualnet 6.0 to analyze the best routing protocol for the Hand over support. The simulation parameters are as given in table 1 and the network scenario as shown in fig 2.
Table 1: Simulation parameters

\begin{tabular}{|c|c|}
\hline Parameters & Value \\
\hline Number of base stations & 2 \\
\hline Number of mobile stations & 4 \\
\hline Frequency & $2.5 \mathrm{GHz}$ \\
\hline Channel bandwidth & $20 \mathrm{MHz}$ \\
\hline Duplexing & TDD \\
\hline Message length & 51200 bytes \\
\hline FTT size & 2048 \\
\hline Maximum power & $50 \mathrm{dBm}$ \\
\hline Transmission power & $20 \mathrm{dBm}$ \\
\hline
\end{tabular}

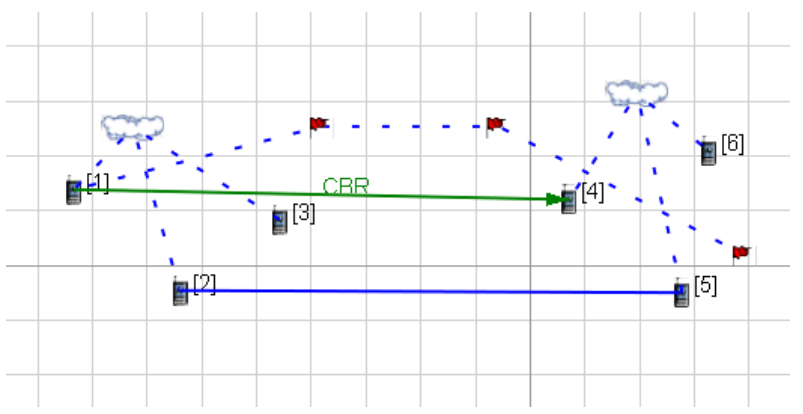

Fig.2 WiMAX simulation scenario

An analysis has been made using different routing protocols for the unicast data sent and the corresponding delay is measured. The amount of data sent by the sending node 1 is 51200 bytes.

Table 2. Comparative table for routing protocols

\begin{tabular}{|c|c|c|}
\hline $\begin{array}{c}\text { Routing } \\
\text { protocol }\end{array}$ & $\begin{array}{c}\text { Unicast data } \\
\text { received(bytes) }\end{array}$ & $\begin{array}{c}\text { End to end } \\
\text { delay(sec) }\end{array}$ \\
\hline AODV & 13,312 & 0.6246 \\
\hline Bellman ford & 9,728 & 0.09631 \\
\hline DYMO & 13,312 & 0.6169 \\
\hline Fisheye & 27,648 & 0.0896 \\
\hline IERP & 51,200 & 9.9024 \\
\hline LANMAR & 27,136 & 0.0732 \\
\hline
\end{tabular}

The above table depicts that LANMAR is the best routing protocol for real time applications i.e. rtPS service class and IERP has been found to be best suited for Best Effort service class.

The following results in Fig 3.1 to 3.6 indicate the amount of data being received in bytes and average end to end delay incurred using different routing protocols for destination node (node id) 4 . 


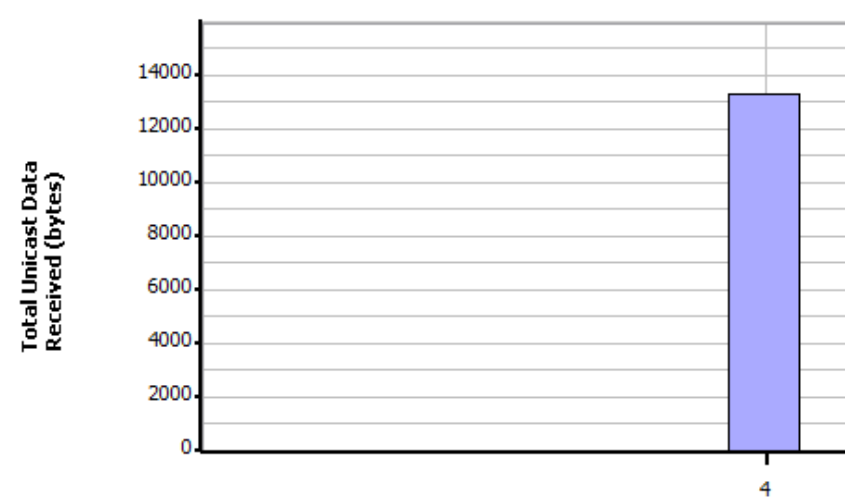

Fig 3.1.a AODV received data13,312 bytes

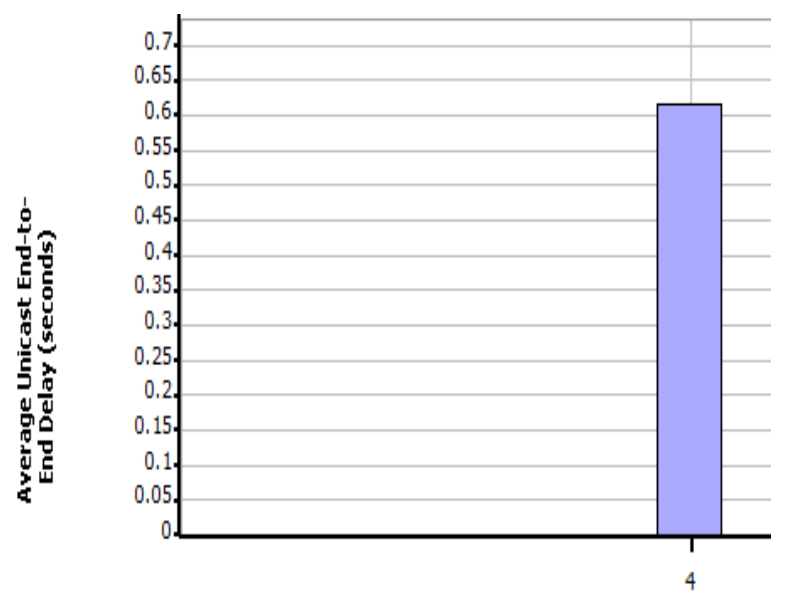

Fig 3.2.a Belmanford data received 9728 bytes

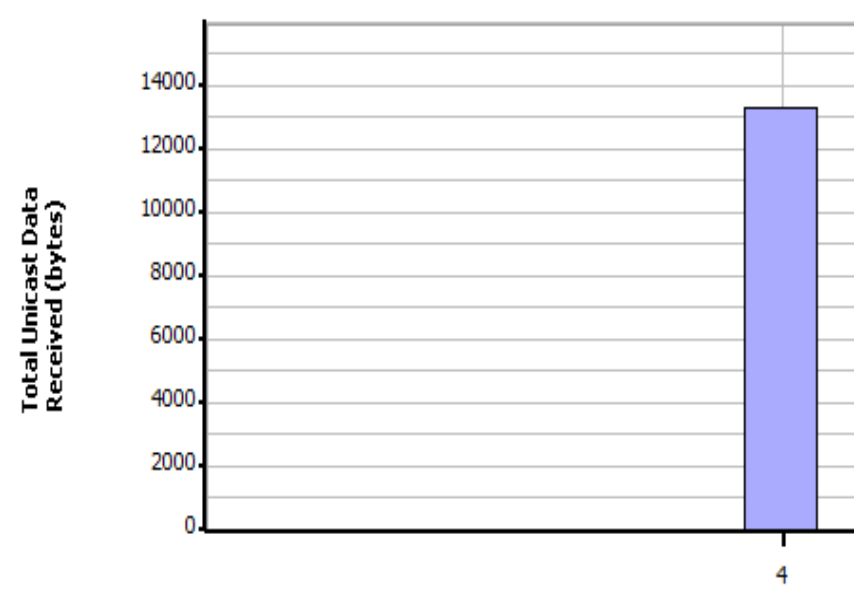

Fig 3.3.a DYMO data received 13,312 bytes

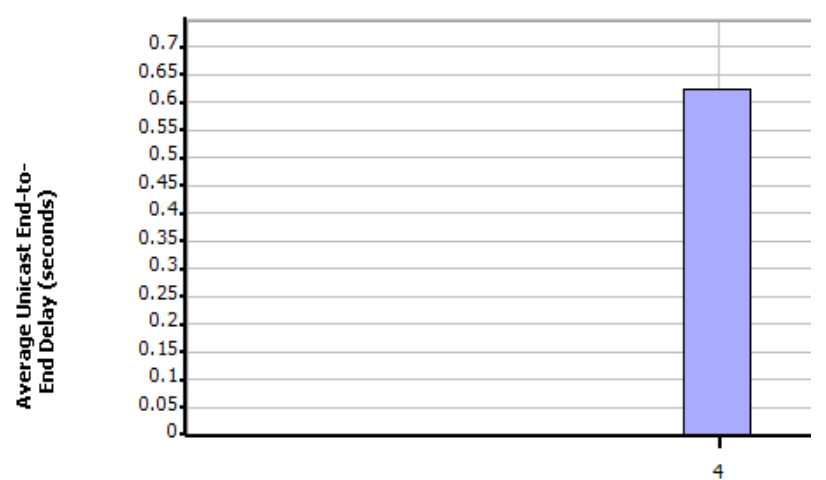

Fig 3.1.b AODV end to end Delay 0.6246 sec

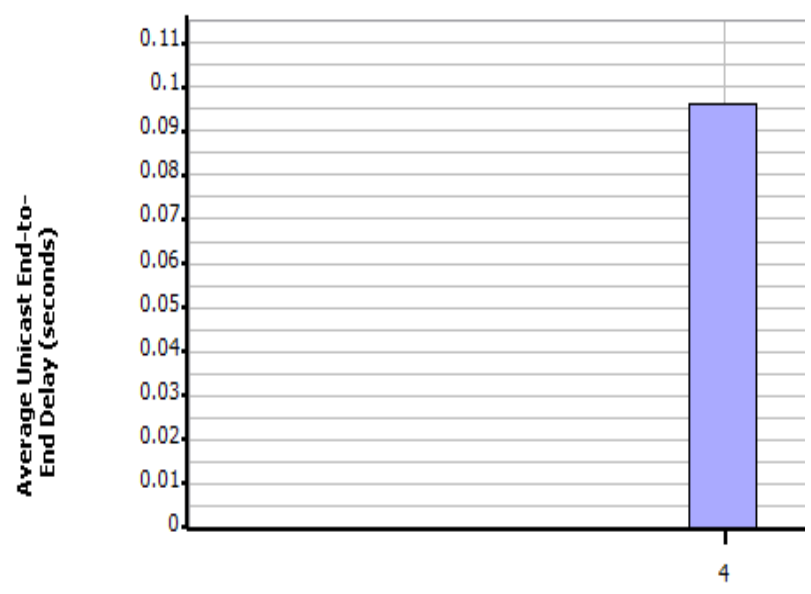

Fig 3.2.b Belmanford Delay 0.09sec

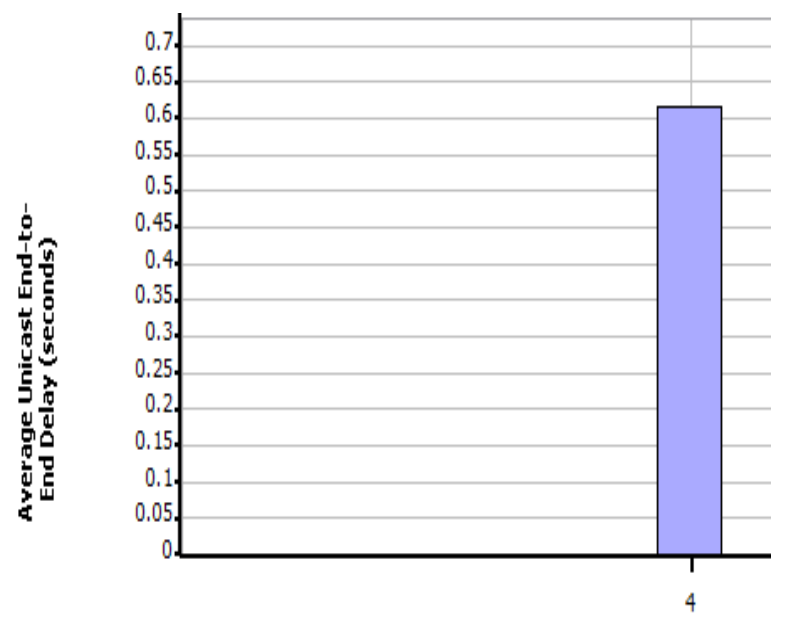

Fig 3.3.b DYMO Delay 0.6169 sec 


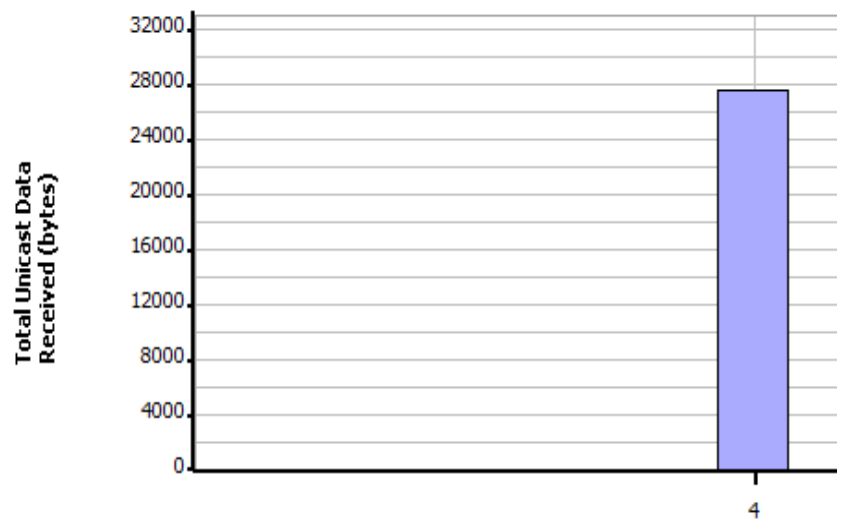

Fig 3.4.a Fisheye data received 27,648 bytes

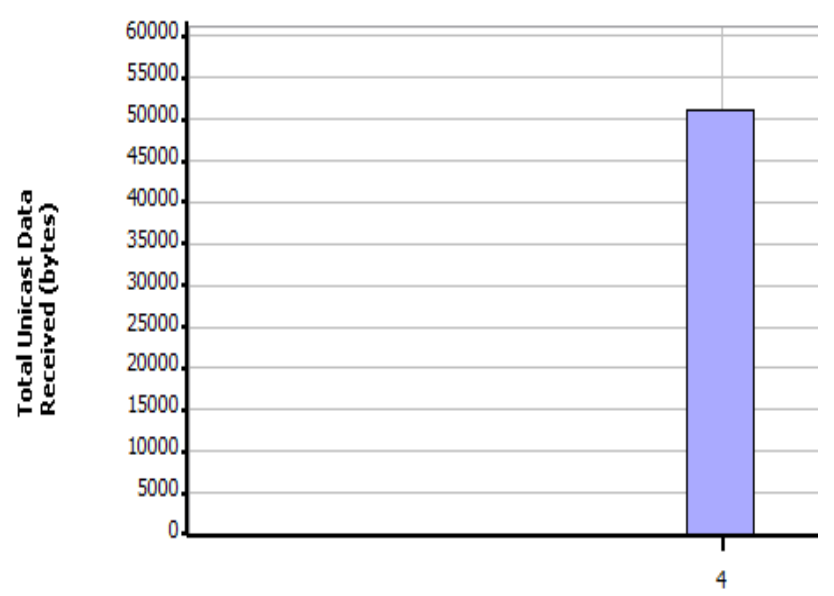

Fig 3.5.a IERP data received 51200 bytes

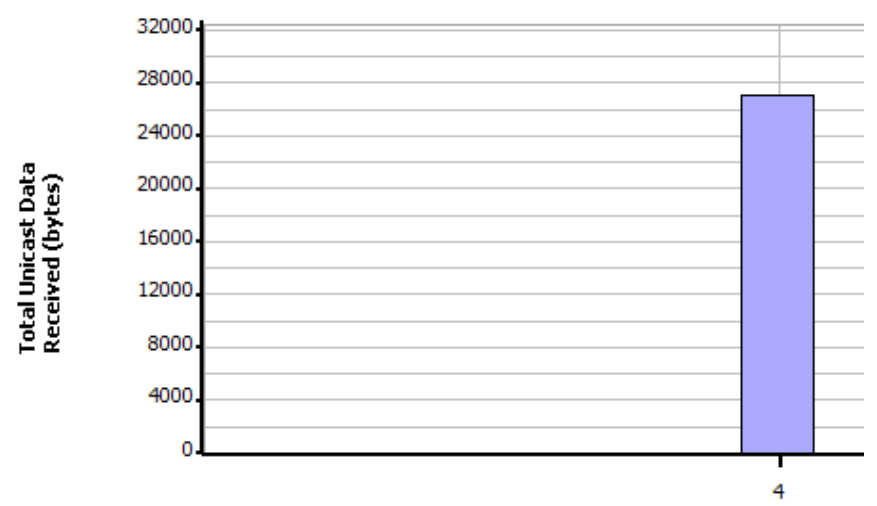

Fig 3.6.a LANMAR data received 27,136

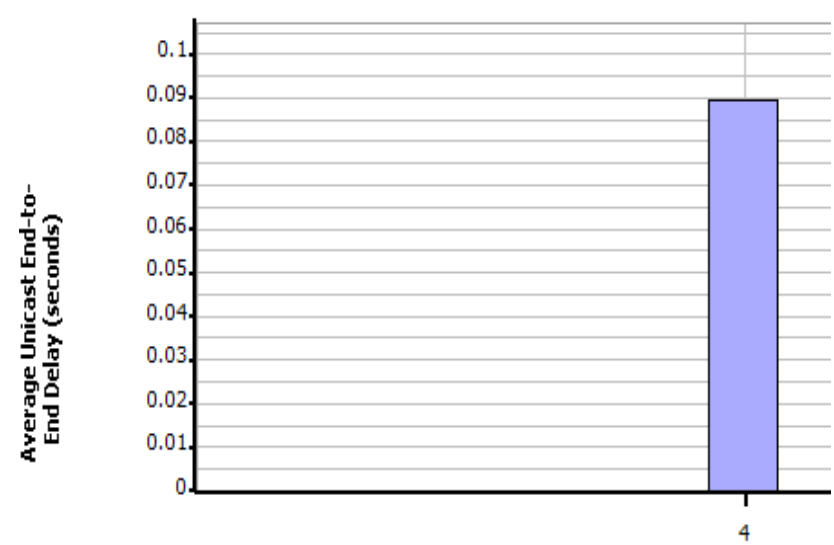

Fig 3.4.b Fisheye Delay $0.0896 \mathrm{sec}$

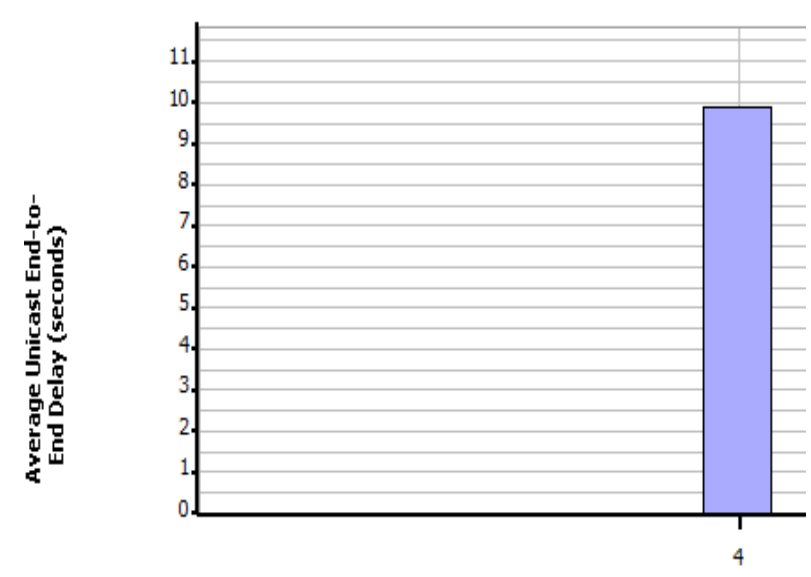

Fig 3.5.b IERP Delay $9.9024 \mathrm{sec}$

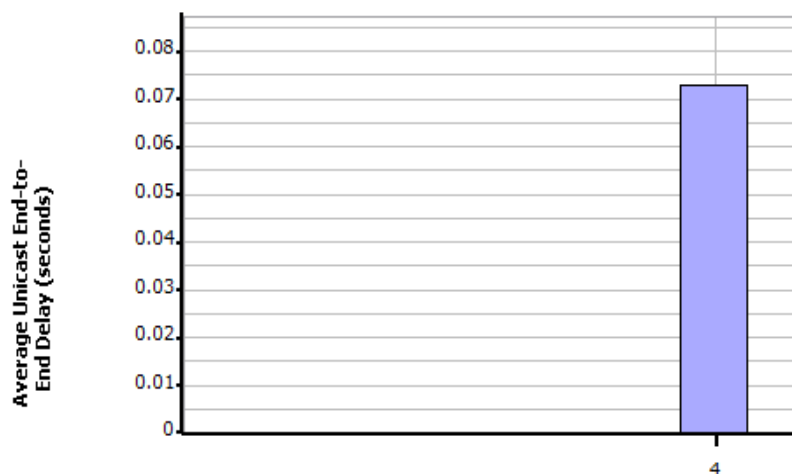

Fig 3.6.b LANMAR 0.073206 sec 


\section{CONCLUSION}

In case of hand over minimization the pre-hand over delay is an important aspect. Furthermore on using the concurrent scanning procedure for the best two target BSs high quality hand over support can be achieved in Mobile WiMAX and problems like ideal sectors, network congestion and fast change in RSS are proposed to be minimized. Fisheye protocol is optimal for smaller network and LANMAR for the larger networks.

\section{ACKNOWLEDGEMENT}

The Authors would like to thank VGST (Vision Group on Science and Technology), Government of Karnataka, India for providing infrastructure facilities to do this project.

\section{REFERENCES}

[1] IEEE Standard 802.16 for Global Broadband Wireless Access http://ieee802.org/16/docs/03/C8021603_14. .

[2] WiMAX Forum Certification of Broadband Wireless Systems, www.WiMAXforum.org.

[3] Mobile WiMAX Part I: A Technical Overview and Performance Evaluation WiMAX forum 2006.

[4] Mobile WiMAX - Part II: A Comparative Analysis, WiMAX Forum, 2006.

[5] Shu Lin \& Daniel J. Costello, Jr. -"Error Control Coding”, Pearson / Prentice Hall, Second Edition, 2004.

[6] David Tse, P. Viswanath, "Fundamentals Of wireless communication", Cambridge 2006.
[7] Bernard Sklar, "Digital Communications: "Fundamentals and Applications, 2nd Edition," January 11, 2001

[8] IEEE 802.16 WG, "Draft IEEE 802.16m System Description Document”, Dec. 2010.

[9] S. K. Ray, S. K. Ray, K Pawlikowski, A.McInnes and H. Sirisena,"Self-Tracking Mobile Station Controls Its Fast Handover in Mobile WiMAX", IEEE Wireless Communications and Research Conference (WCNC), Sydney, Australia, 18-21 April 2010.

[10] J. Lee, H. Kim, and K. Kim, "Resource reservation and allocation based on direction prediction for handoff in mobile multimedia. Networks", Computational Science-ICCS 2003, pp. 719-720, 2003.

[11] "Movement Direction-based Handover Scanning for Mobile WiMAX" by Mohammed A.Ben- Mubarak, Borhanuddin Mohd. Ali, Nor K. Noordin, 2011, 17th Asia-Pacific Conference on Communications (APCC) 2nd - 5th October 2011 | Sutera Harbour Resort, Kota Kinabalu, Sabah, Malaysia.

[12] M. Ben-Mubarak, B.M. Ali, N.K. Noordin, A.Ismail and C.K. Ng, "Review of Handover to Support Mechanisms Triple Play in Mobile WiMAX", IETE Tech Rev, pp. 258-267, 2009

[13] IEEE Standard for Local and metropolitan area networks Part 16: Air Interface for Fixed and Mobile Broadband Wireless Access Systems, 2006. 